\title{
Strategies for a Computer-Aided Discovery of Novel Open-Shell Polymers
}

\author{
Omri D. Abarbanel, ${ }^{\dagger}$ Julisa Rozon, ${ }^{\dagger}$ and Geoffrey R. Hutchison ${ }^{*, \dagger, \ddagger}$ \\ †Department of Chemistry, University of Pittsburgh, 219 Parkman Avenue, Pittsburgh, \\ Pennsylvania 15260, United States \\ $\ddagger$ Department of Chemical and Petroleum Engineering, University of Pittsburgh, 3700 \\ O'Hara Street, Pittsburgh, Pennsylvania 15261, United States \\ E-mail: geoffh@pitt.edu
}




\begin{abstract}
Organic $\pi$-conjugated polymers with a triplet ground state have been the focus of recent research for their interesting and unique electronic properties, arising from the presence of the two unpaired electrons. These polymers are usually built from alternating electron-donating and electron-accepting monomer pairs which lower the HOMO-LUMO gap and yield a triplet state instead of the typical singlet ground state. In this paper we use density functional theory calculations to explore the design rules that govern the creation of a ground state triplet conjugated polymer, and find that a small HOMO-LUMO gap in the singlet state is the best predictor for the existence of a triplet ground state, compared to previous use of pro-quinoidal character. This work can accelerate the discovery of new stable triplet materials by reducing the computational resources needed for electronic-state calculations and the number of potential candidates for synthesis.
\end{abstract}

\title{
Graphical TOC Entry
}

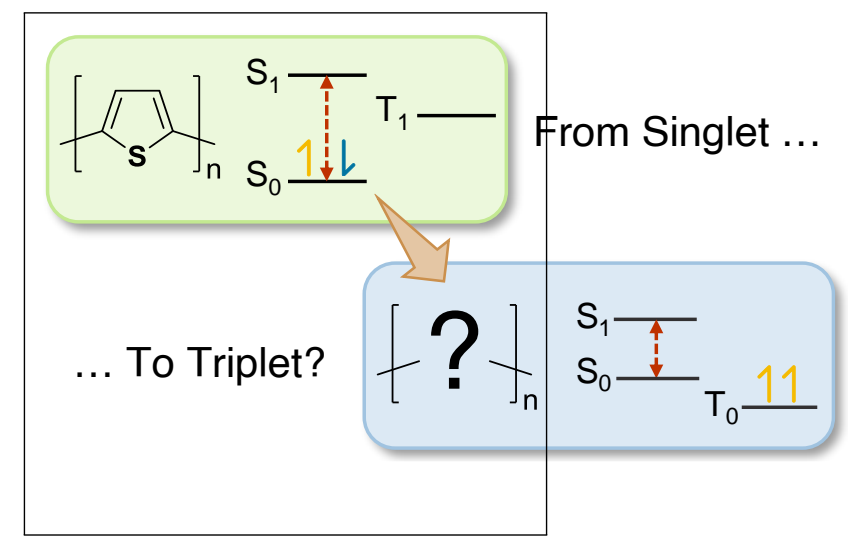

\section{Keywords}

Conjugated Polymers, Triplet Ground State, Computational Chemistry 
Organic $\pi$-conjugated polymers have been a focus of fundamental research for many years thanks to their delocalized electronic properties, which can be used in a wide variety of electronic applications. ${ }^{1,2}$ The ground state of the vast majority of those polymers is a singlet state $\left(\mathrm{S}_{0}\right)$, which can be excited to a triplet $\left(\mathrm{T}_{1}\right)$ state via different pathways, such as intersystem crossing or thermally-activated delayed fluorescence. ${ }^{3-5}$ However, in recent years organic $\pi$-conjugated polymers with a triplet ground state (dubbed " $\mathrm{T}_{0}$ ") have been discovered and studied for their unique electronic, optical, and magnetic properties arising from their unpaired electrons. Such ground-state triplet materials have found applications as varied as batteries, ${ }^{6}$ supercapacitors,${ }^{7}$ non-linear optics, ${ }^{8}$ and many others. ${ }^{9-12}$

Understanding the design rules for the synthesis of such molecular diradicals can aid with the discovery of new materials. Those structure/function correlations can help us determine the type of monomers that will promote a triplet ground state, how the electronic structure is affected, and how to design new materials. For example, previous studies have used "proquinoidal" monomers in order to create high-spin polymers, suggesting that a quinoidal bonding character helps to stabilize the diradical polymer. ${ }^{12-15}$ However, others challenge this, by suggesting instead that aromatic bonding character stabilizes the diradical ground state. ${ }^{10}$ These two opposing hypotheses can lead to different design rules, but by finding the best predictors of a stable ground-state triplet, we can assist in the discovery process.

In this work, we have used dispersion-corrected $\omega$ B97X-D3 density functional theory (DFT) method to calculate the ground state energies of both singlet and triplet states of various $\pi$-conjugated oligomers. Our data set consists of 11 donor monomers and 12 acceptor monomers (Figure 1), most previously studied by the Azoulay group, ${ }^{7,16-20}$ yielding a set of 132 oligomers. The geometry optimization steps, the single-point electronic energy calculations, and the calculation of $\Delta E_{T-S}$ were done as described in the Computational Methods section below.

To find key predictors of ground state triplets, we compared the a variety of electronic and 
geometric properties as predictors of the energy difference between the triplet and singlet energies. In addition, we considered different strategies to favor triplet stabilization, such as changing the heteroatom in the polymer backbone, and forcing quinoidal bonding character. This work can aid in the discovery of new and novel open shell materials by increasing the search speed and decreasing the search space of potential candidates.

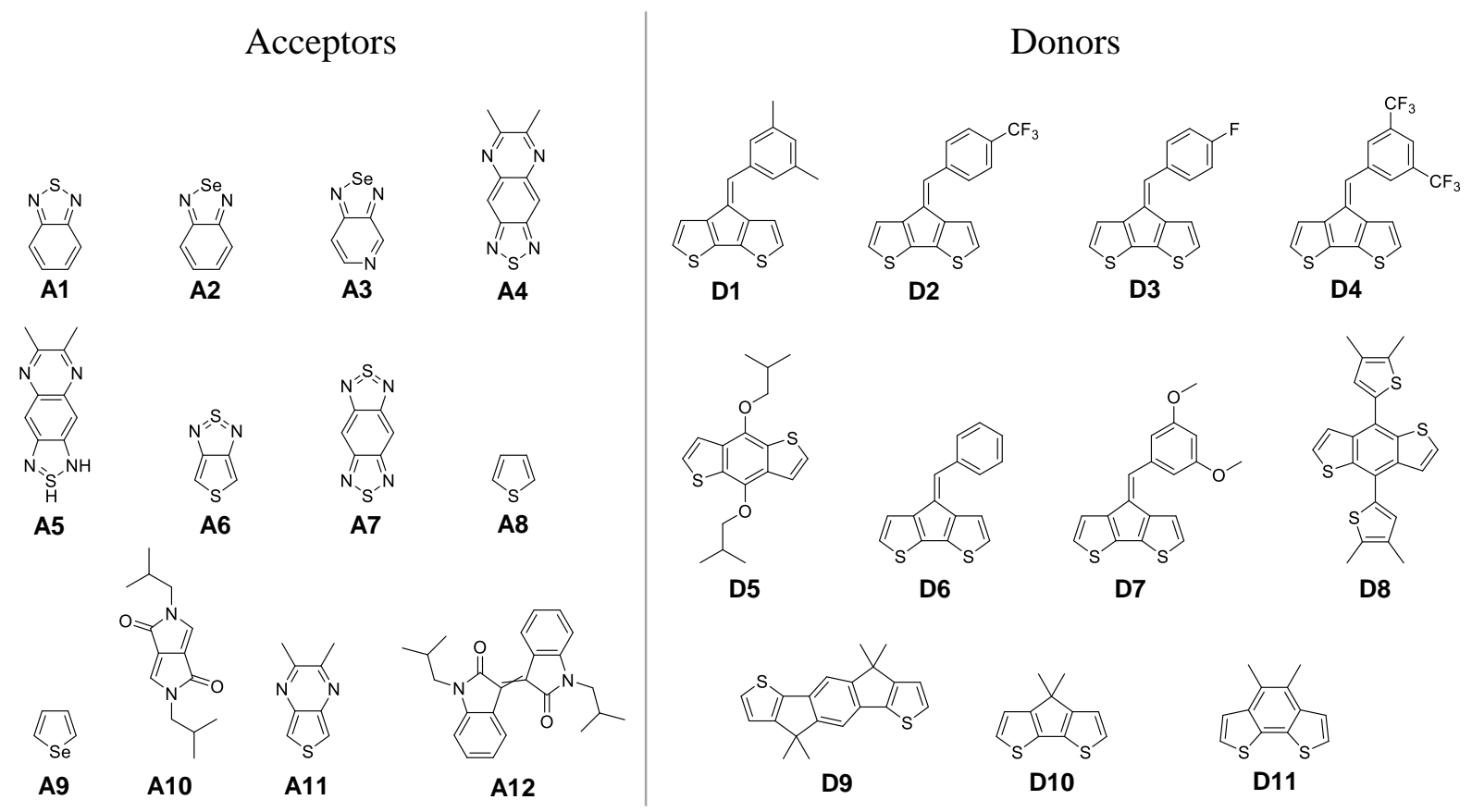

Figure 1: The acceptors and donors used to create the tetramers.

As mentioned, one of the working hypotheses for the stability of a triplet ground-state is through a bi-radical system, in which each unpaired electron is in different singly-occupied molecular orbitals (SUMO), and the formation of a semi-quinoidal bonding pattern in the polymer backbone. ${ }^{11,13,14}$ This suggests that the bond between the monomers should have some double-bond character — and thus be shorter to stabilize the triplet ground-state. We measured the inter-monomer bond length of the oligomers as a metric of quinoidal character, and compared this geometric measure to the difference between the electronic energies of the triplet and singlet species $\Delta E_{T-S}$ (Figure 2). 
(a)

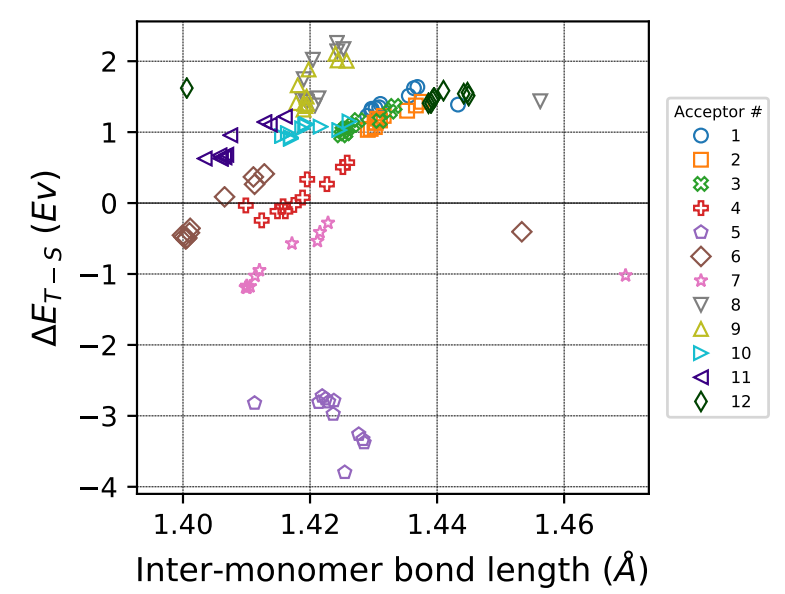

(b)

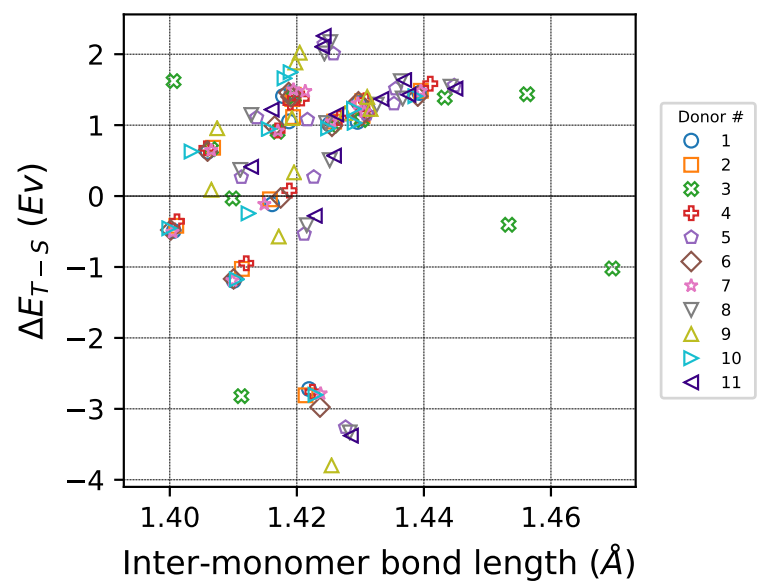

Figure 2: Correlation between $\Delta E_{T-S}$, in $\mathrm{eV}$, and the inter-monomer bond length, in $\AA$, grouped by (a) acceptor number and (b) donor number.

Our calculations, however, show very little correlation between the inter-monomer bond length and the stability of the triplet ground state across all 132 oligomers. While there is a weak trend for some families of oligomers that share the same acceptor (Figure 2(a)), there are a few exceptions. In some cases, such as for the oligomer of donor D3 and acceptor A7, while it had a stable triple ground state - its calculated inter-monomer bond length was the longest at $1.47 \AA$. In contrast, the oligomer of donor D3 and A12 does not yield a stable triplet ground state, but has one of the shortest calculated inter-monomer bond lengths at $1.40 \AA$.

Furthermore, oligomers with acceptor A5 yield a negative slope between the inter-monomer bond length and $\Delta E_{T-S}$, while all other families have a positive slope (Table $\mathrm{S} 1$ ). Thus, while looking at some specific acceptors we can see a trend, there is no overall correlation between the stability of the triplet ground state and the inter-monomer bond length. In addition, there is no correlation between the inter-monomer length and the donor number (Figure 2(b)).

While the pro-quinoidal design rule might work for specific cases, the stability of the triplet 
ground state instead comes from a broader property — narrowing of the singlet HOMOLUMO gap. While it has been shown that one strategy to lower the HOMO-LUMO gap is by quinoidal bonding, ${ }^{21,22}$ it is by far not the only design rule.

A small HOMO-LUMO gap has been shown to promote a lower triplet energy level, increasing the likelihood of a high-spin ground state as the frontier molecular orbitals (MO) become closer energetically. ${ }^{14,23,24}$ We therefore compared the energy difference between the singlet and triplet of each oligomer $\left(\Delta E_{T-S}\right)$ versus the HOMO-LUMO gap of the corresponding singlet-state oligomer, both in eV (Figure 3). Figures 3(a) and 3(b) show the same correlation and only differ by grouping of the acceptor number and donor number, respectively. Out of 132 oligomers, 35 had ground-state triplet states, based on the optimized geometries and $\omega$ B97X-D3/def2-SVP single-point energies. A significant correlation between those energies can be seen, with a correlation of determination $\left(R^{2}\right)$ of 0.96 and a linear relation with an $\mathrm{x}$-axis intercept at $3.84 \mathrm{eV}$ (Eq. S1).

(a)

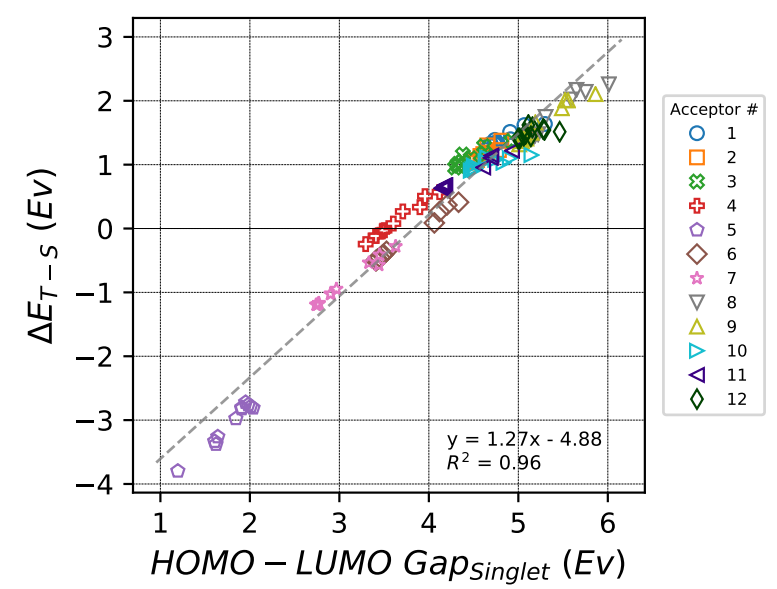

(b)

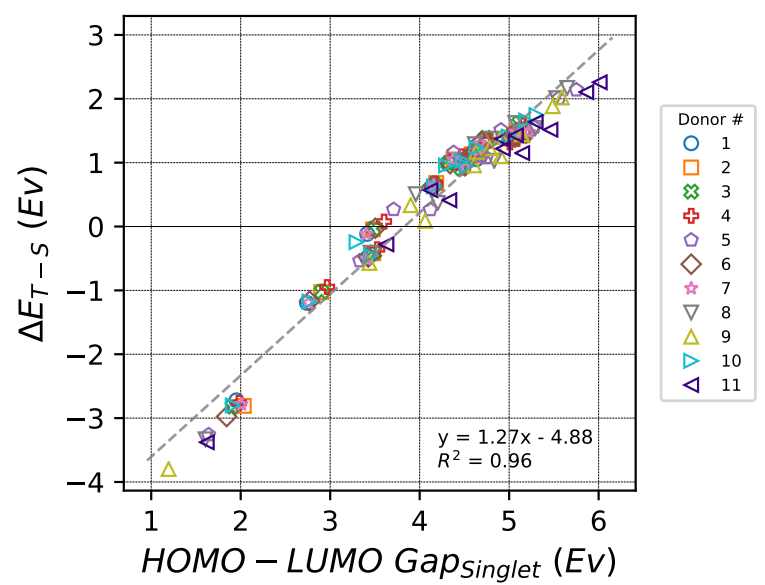

Figure 3: Correlation plots between the difference of the Triplet and Singlet energies of each oligomer versus its the HOMO-LUMO gap of the singlet species, both in eV, grouped by (a) the acceptor number and (b) the donor number. Linear best-fit line is shown as a dashed gray line.

As seen in Figure 3(b), there is very little correlation between the donor identity and $\Delta E_{T-S}$. 
However, from Figure 3(a) it can be seen that oligomers that share the same acceptor monomer are grouped in close proximity, indicating that a high-spin system is strongly dependant on the identity of the acceptor.

From this correlation we can see that acceptor A5 appears to have the lowest singlet HOMOLUMO gap and the most stable triplet state, followed by acceptor A7. Acceptors A4 and A6 both have several oligomers with stable triplet ground states; six oligomers for acceptor A4 and eight oligomers for acceptor A6. Interestingly, acceptor A5, which is the hydrogenated version of acceptor A4, was possibly made due to a human error. Nonetheless, this unintentional discovery resulted in a monomer that promoted a stable ground state triplet. However, we acknowledge that, realistically, the synthesis of acceptor A5 might prove to be a difficult endeavor.

In addition, we have examined the correlation between the singlet HOMO-LUMO gap calculated using the $\omega$ B97X-D DFT method and the GFN2-xTB semi-empirical method (Figure S1). Oligomers that contain acceptor A5 are outliers, showing no correlation with the rest of the set. Due to the low synthetic viability of acceptor A5, we removed those oligomers from the comparison. We fit the data points to linear, logarithmic, and radical functions, and found that the logarithmic function has the highest $R^{2}$ at 0.96 , compared to 0.89 and 0.94 for the linear and square root functions, respectively. In short, while the HOMO-LUMO eigenvalues from density functional theory are unphysical, and gaps from $\omega$ B97X-D may be too large, and from GFN2-xTB may be too small compared to experiment, there is still a strong correlation between the two computational methods. This correlation can thus be used in the discovery of new ground state triplet oligomers, as only a single semi-empirical calculation is needed, skipping multiple time-consuming DFT calculations.

To further consider why the stability of a triplet ground state is dependent on the acceptor identity, the HOMO and LUMO eigenvalues of each acceptor and donor monomers were calculated, following the same process as the oligomers, as described in the Methods section 
(Figure S2). The HOMO eigenvalues on the donor monomers are, of course, generally less negative (above $-8 \mathrm{eV}$ ) than the HOMO energies of the acceptor monomers (below $-8 \mathrm{eV}$, with the exception of acceptors A5, A10, and A12.) Acceptors A4, A6, and A7, which yielded some oligomers with ground-state triplets, have similar HOMO energies at $-8.30 \mathrm{eV}$, $-8.34 \mathrm{eV}$, and $-8.34 \mathrm{eV}$ respectively. Acceptor A11 has a similar HOMO eigenvalue, at -8.32 $\mathrm{eV}$, but does not show triplet ground-state stability.

Acceptor A5 is the exception in this case, as it has a relatively high HOMO eigenvalue, at $-6.66 \mathrm{eV}$ - higher than all the other acceptor and donor monomers. This suggests that acceptor A5 might act as a very good donor. It would be interesting, to find other synthetically-accessible monomers with similar HOMO and LUMO eigenvalues and compare their performance to acceptor A5. However, this is beyond the scope of this work.

As we have shown, the most reliable predictor for the stability of the triplet ground state is the HOMO-LUMO gap of the singlet species. We therefore considered different general strategies to lower gap. Previous studies have shown that the identity of the heteroatom in the backbone of an oligomer as well as the identity of the side group affect the HOMO-LUMO energy gap. ${ }^{25,26}$ We chose sulfur (S), nitrogen (N) and selenium (Se) as the representative heteroatom of a 5-membered aromatic heterocycle; since we expected the HOMO-LUMO gap of the oligomer to decrease as the HOMO-LUMO gap of the heteroatom decreases. ${ }^{25,27}$ The side group, always on the 3- and 4- position in the heteroycle, representatives are a fused benzene ring for its potentially stabilizing effect on the quinoidal form, ${ }^{28}$ an ethylenedioxy group for its electron-donating effects, ${ }^{29}$ and no side group as a reference.

This set consists of 12 monomers - pyrrole, thiophene, selenophene, their 3-4-ethylenedioxyderivatives (EDOP, EDOT, and EDOS respectively) and their benzo- derivatives (BP, BT, and BS respectively). In addition, we constructed a quinoidal version of the thiophene-based oligomers by forcing inter-monomer double bonds by adding methylidene $\left(\mathrm{H}_{2} \mathrm{C}=\right)$ terminating groups (q-Thiophene, q-EDOT, and q-BT respectively), as can be seen in Figure 4(a). 
By forcing a quinoidal bonding structure we can have a straightforward comparison with the aromatic bonding structure. We constructed the hexamer of each system, as previous studies show a high correlation with the calculated electronic energies of longer oligomers, ${ }^{30,31}$ and followed the same geometry optimizations and single-point calculations of both singlet and triplet species as described in the Computational Methods section below.

(a)

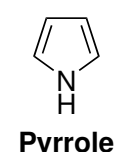

Pyrrole

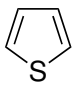

Thiophene

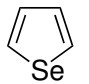

Selenophene

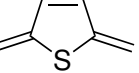

q-Thiophene

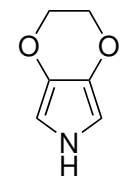

EDOP

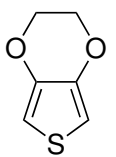

EDOT

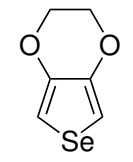

EDOS

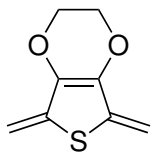

q-EDOT
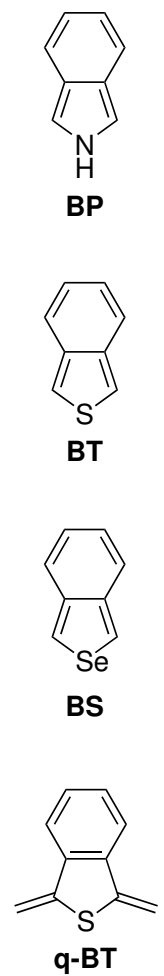

(b)

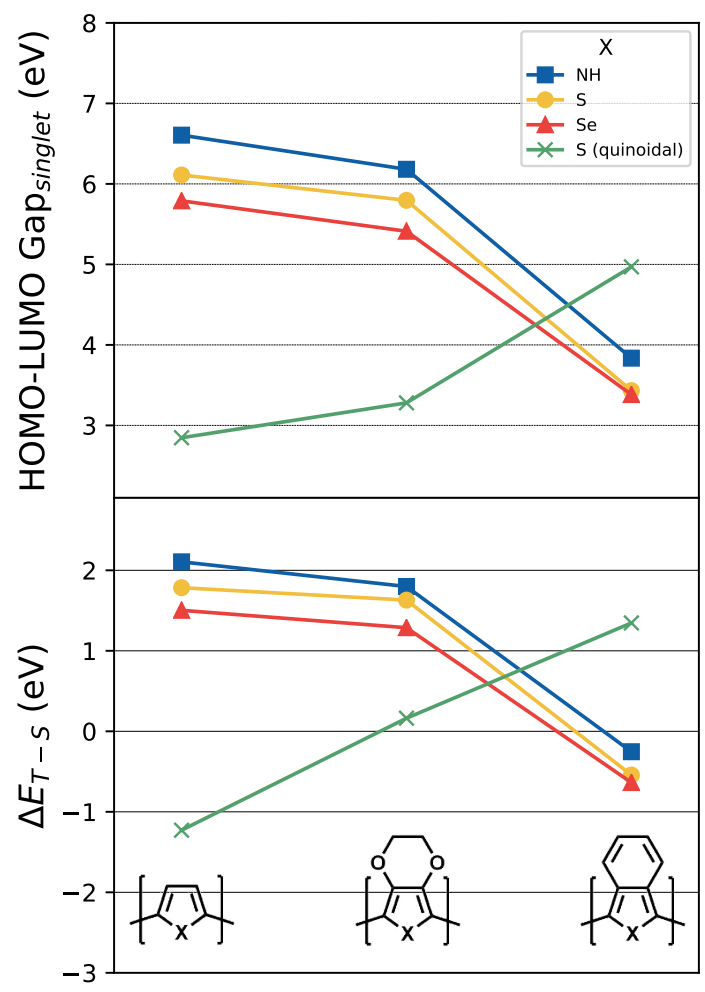

Figure 4: (a) The 12 monomers used in finding a strategy to lower the HOMOLUMO gap - Pyrrol, 3-4-Ethyldioxypyrrole (EDOP), Benzopyrrole (BP), Thiophene, 3-4-Ethyldioxythiophene (EDOT), Benzothiophene (BT), Selenophene, 3-4Ethyldioxyselenophene (EDOS), Benzoselenophene (BS), and the quinoidal versions of the thiophene-based monomers - denoted with "q-", (b) The singlet HOMO-LUMO gap of the hexamers (on the top), and their $\Delta E_{T-S}$ (on the bottom), both in $\mathrm{eV}$.

Figure 4(b), demonstrates that lower singlet HOMO-LUMO gap correlates with a lower $\Delta E_{T-S}$ and a more stable triplet ground state. As expected, we also see correlation between the singlet HOMO-LUMO gap energy of the heteroatom and the singlet HOMO-LUMO gap energy of the hexamer, where nitrogen yields a higher gap, followed by sulfur, and lastly 
selenium with the lowest gap, consistent with previous results.

However, the side group identity has a large effect on the HOMO-LUMO gap energy as well. Figure 4b illustrates how the benzo- derivatives have a significantly lower HOMO-LUMO gap energy than the ethylenedioxy- derivatives, which itself has only a moderate reduction of the HOMO-LUMO gap energy over the parent oligomers with no side group.

In contrast to the findings above, the forced-quinoidal form of the thiophene-based oligomers show an opposite trend compared to its aromatic counterpart. While the quinoidal form of thiophene hexamer has a significantly lower HOMO-LUMO gap than the aromatic thiophene hexamer and a more moderate reduction compared to the ethylenedioxy- derivatives, this relationship reverses when it comes to the benzo- derivatives. While some promote a quinoidal bonding scheme as a strategy to induce a triplet ground state, ${ }^{11,13,14}$ we show here that this is not the case. This conclusion reinforces the discussion above, that a pro-quinoidal molecular design is guaranteed to make a polymer with a stable tripled ground state. The best overall strategy is to use monomers that promote a lower HOMO-LUMO gap, either by conjugation or inductively.

In conclusion, polymers with a triplet ground state have unusual and interesting properties that can open the door to novel and exciting applications. In this study we considered various strategies to produce such materials. We examined the correlation between the calculated stability of oligomers with a triplet ground state versus various electronic and geometric properties. We have found a high correlation between the energy difference between the triplet and singlet states of the oligomer $\left(\Delta E_{T-S}\right)$ and the HOMO-LUMO gap of the singlet state. We can use this correlation in order to find new candidates with triplet character by calculating just the HOMO-LUMO gap of the singlet. Moreover, general strategies to produce low band gap $\pi$-conjugated polymers can be used to find novel ground-state triplet materials.

While pro-quinoidal design strategies may yield ground-state triplet polymers, our exami- 
nation over 132 oligomers suggests there is no overall correlation between quinoidal bond character and the stability of the triplet state. In addition, we have found that the HOMO and LUMO energies of the donors and acceptor monomers are also poor predictors for the stability of the triplet ground state. Instead, heteroatom substitution (e.g., sulfur to selenium) and side-group substitutions appear to yield increased stability of the triplet state without necessarily inducing quinoidal character.

These design rules can, in turn, be used for both experimental and computational design of new ground-state triplet conjugated materials. For example, use of a genetic algorithm or other generative method could sample a large number of potential oligomers through computational design.

\section{Computational Methods}

As mentioned above, the data set consists of 11 donor monomers and 12 acceptor monomers (Figure 1) studied by the Azoulay group. ${ }^{7,16-20}$ Long alkyl chains were replaced with shorter methyl groups in order to reduce computational needs with negligible effect on the electronic properties. A tetramer (octamer in Azulay's notation) of every possible donor-acceptor pair (i.e. DADADADA, where $\mathrm{D}=$ donor monomer and $\mathrm{A}=$ acceptor monomer) was created from their respective Simplified molecular-input line-entry system (SMILES) string, ${ }^{32-34}$ giving a set of 132 oligomers.

The geometry optimization of every oligomer was done in steps in order to reduce computational costs, starting with a conformer search and optimization using MMFF94 ${ }^{35}$ or $\mathrm{UFF}^{36}$ with OpenBabel version 3.1.0, ${ }^{37}$ followed by GFN2-xTB, ${ }^{38}$ and ending with the B97-3c DFT functional ${ }^{39}$ using Orca version $4.2 .0 .{ }^{40,41}$

The single-point energy of each oligomer was calculated on the final optimized B97-3c geometry using the dispersion-corrected $\omega$ B97X-D3 DFT functional ${ }^{42}$ with the def2-SVP basis 
set $^{43}$ using Orca. This process was done for both singlet and triplet species of each oligomer. The output files were processed using Python with the cclib library ${ }^{44}$ for the electronic, highest occupied molecular orbital (HOMO) and lowest unoccupied molecular orbital (LUMO) eigenvalues, and the Open Babel library $^{37}$ for the extraction of the inter-monomer bond lengths. The difference between the triplet and singlet energies was calculated as

$$
\Delta E_{T-S}=E_{\text {Triplet }}-E_{\text {Singlet }}
$$

\section{Acknowledgement}

We acknowledge support from Department of Energy-Basic Energy Sciences Computational and Theoretical Chemistry (Award DE-SC0019335) and the University of Pittsburgh Center for Research Computing through the computational resources provided.

\section{Supporting Information Available}

See Supplementary Information for additional figures and data. A repository with all raw data, Python notebooks, and the SMILES strings of the monomers is available at https: //github.com/hutchisonlab/GST

\section{References}

(1) Facchetti, A. $\pi$-Conjugated Polymers for Organic Electronics and Photovoltaic Cell Applications $\dagger$. Chemistry of Materials 2010, 23, 733-758.

(2) Tadesse, T. Application of Conjugated Organic Polymers for Photovoltaic's: Review. 2018,

(3) Liu, Y.; Hua, L.; Yan, S.; Ren, Z. Halogenated $\pi$-conjugated polymeric emitters with 
thermally activated delayed fluorescence for highly efficient polymer light emitting diodes. Nano Energy 2020, 73, 104800.

(4) Liu, Y.; Yan, S.; Ren, Z. $\pi$-Conjugated polymeric light emitting diodes with sky-blue emission by employing thermally activated delayed fluorescence mechanism. Chemical Engineering Journal 2021, 417, 128089.

(5) Rao, J.; Yang, L.; Li, X.; Zhao, L.; Wang, S.; Tian, H.; Ding, J.; Wang, L. StericallyLocked Donor-Acceptor Conjugated Polymers Showing Efficient Thermally Activated Delayed Fluorescence. Angewandte Chemie International Edition 2021, 60, 9635-9641.

(6) Morita, Y.; Nishida, S.; Murata, T.; Moriguchi, M.; Ueda, A.; Satoh, M.; Arifuku, K.; Sato, K.; Takui, T. Organic tailored batteries materials using stable openshell molecules with degenerate frontier orbitals. Nature Materials 2011 10:12 2011, 10, 947-951.

(7) Wang, K.; Huang, L.; Eedugurala, N.; Zhang, S.; Sabuj, M. A.; Rai, N.; Gu, X.; Azoulay, J. D.; Ng, T. N. Wide Potential Window Supercapacitors Using Open-Shell Donor-Acceptor Conjugated Polymers with Stable N-Doped States. Advanced Energy Materials 2019, 9, 1902806.

(8) Nakano, M. Open-Shell-Character-Based Molecular Design Principles: Applications to Nonlinear Optics and Singlet Fission. Chemical Record 2017, 17, 27-62.

(9) Sun, Z.; Ye, Q.; Chi, C.; Wu, J. Low band gap polycyclic hydrocarbons: from closedshell near infrared dyes and semiconductors to open-shell radicals. Chemical Society Reviews 2012, 41, 7857-7889.

(10) Huang, Y.; Egap, E. Open-shell organic semiconductors: an emerging class of materials with novel properties. Polymer Journal 2018 50:8 2018, 50, 603-614. 
(11) Huang, L.; Eedugurala, N.; Benasco, A.; Zhang, S.; Mayer, K. S.; Adams, D. J.; Fowler, B.; Lockart, M. M.; Saghayezhian, M.; Tahir, H. et al. Open-Shell DonorAcceptor Conjugated Polymers with High Electrical Conductivity. 2020,

(12) Tam, T. L. D.; Wu, G.; Chien, S. W.; Lim, S. F. V.; Yang, S. W.; Xu, J. High Spin ProQuinoid Benzo[1,2-c;4,5-c']bisthiadiazole Conjugated Polymers for High-Performance Solution-Processable Polymer Thermoelectrics. ACS Materials Letters 2020, 2, 147152.

(13) Ji, X.; Fang, L. Quinoidal Conjugated Polymers with Open-Shell Characters. Polymer Chemistry 2021,

(14) London, A. E.; Chen, H.; Sabuj, M. A.; Tropp, J.; Saghayezhian, M.; Eedugurala, N.; Zhang, B. A.; Liu, Y.; Gu, X.; Wong, B. M. et al. A high-spin ground-state donoracceptor conjugated polymer. Science Advances 2019, 5, eaav2336.

(15) Dexter Tam, T. L.; Ng, C. K.; Lim, S. L.; Yildirim, E.; Ko, J.; Leong, W. L.; Yang, S. W.; Xu, J. Proquinoidal-conjugated polymer as an effective strategy for the enhancement of electrical conductivity and thermoelectric properties. Chemistry of Materials 2019, 31, 8543-8550.

(16) Wu, Z.; Yao, W.; London, A. E.; Azoulay, J. D.; Ng, T. N. Temperature-Dependent Detectivity of Near-Infrared Organic Bulk Heterojunction Photodiodes. ACS Applied Materials and Interfaces 2017, 9, 1654-1660.

(17) Sulas, D. B.; London, A. E.; Huang, L.; Xu, L.; Wu, Z.; Ng, T. N.; Wong, B. M.; Schlenker, C. W.; Azoulay, J. D.; Sfeir, M. Y. Preferential Charge Generation at Aggregate Sites in Narrow Band Gap Infrared Photoresponsive Polymer Semiconductors. Advanced Optical Materials 2018, 6, 1701138.

(18) Joo, Y.; Huang, L.; Eedugurala, N.; London, A. E.; Kumar, A.; Wong, B. M.; Boudouris, B. W.; Azoulay, J. D. Thermoelectric Performance of an Open- 
Shell Donor-Acceptor Conjugated Polymer Doped with a Radical-Containing Small Molecule. Macromolecules 2018, 51, 3886-3894.

(19) Wu, Z.; Zhai, Y.; Kim, H.; Azoulay, J. D.; Ng, T. N. Emerging Design and Characterization Guidelines for Polymer-Based Infrared Photodetectors. Accounts of Chemical Research 2018, 51, 3144-3153.

(20) Zhang, S.; Ocheje, M. U.; Huang, L.; Galuska, L.; Cao, Z.; Luo, S.; Cheng, Y.-H.; Ehlenberg, D.; Goodman, R. B.; Zhou, D. et al. The Critical Role of Electron-Donating Thiophene Groups on the Mechanical and Thermal Properties of Donor-Acceptor Semiconducting Polymers. Advanced Electronic Materials 2019, 5, 1800899.

(21) Asaduzzaman, A. M.; Schmidt-D’Aloisio, K.; Dong, Y.; Springborg, M. Properties of polythiophene and related conjugated polymers: a density-functional study. Phys. Chem. Chem. Phys. 2005, 7, 2714-2722.

(22) Huang, J.; Lu, S.; Chen, P.-A.; Wang, K.; Hu, Y.; Liang, Y.; Wang, M.; Reichmanis, E. Rational Design of a Narrow-Bandgap Conjugated Polymer Using the Quinoidal Thieno[3,2-b]thiophene-Based Building Block for Organic Field-Effect Transistor Applications. Macromolecules 2019, 52, 4749-4756.

(23) Salem, L.; Rowland, C. The Electronic Properties of Diradicals. Angewandte Chemie International Edition in English 1972, 11, 92-111.

(24) Gopalakrishna, T. Y.; Zeng, W.; Lu, X.; Wu, J. From open-shell singlet diradicaloids to polyradicaloids. Chemical Communications 2018, 54, 2186-2199.

(25) Kaloni, T. P.; Schreckenbach, G.; Freund, M. S. Band gap modulation in polythiophene and polypyrrole-based systems. Scientific Reports 2016, 6, 36554 .

(26) Bouzzine, S. M.; Salgado-Morán, G.; Hamidi, M.; Bouachrine, M.; Pacheco, A. G.; 
Glossman-Mitnik, D. DFT Study of Polythiophene Energy Band Gap and Substitution Effects. 2015,

(27) JOHNSON III,; RUSSELL, D. NIST Computational Chemistry Comparison and Benchmark Database. 2020.

(28) Yamamoto, K.; Ie, Y.; Nitani, M.; Tohnai, N.; Kakiuchi, F.; Zhang, K.; Pisula, W.; Asadi, K.; Blom, P. W. M.; Aso, Y. Oligothiophene quinoids containing a benzo[c]thiophene unit for the stabilization of the quinoidal electronic structure. $J$. Mater. Chem. C 2018, 6, 7493-7500.

(29) Singh, S. K.; Crispin, X.; Zozoulenko, I. V. Oxygen Reduction Reaction in Conducting Polymer PEDOT: Density Functional Theory Study. The Journal of Physical Chemistry C 2017, 121, 12270-12277.

(30) Abarbanel, O. D.; Hutchison, G. R. Machine learning to accelerate screening for Marcus reorganization energies. Journal of Chemical Physics 2021, 155, 54106.

(31) Zade, S. S.; Bendikov, M. From Oligomers to Polymer: Convergence in the HOMOLUMO Gaps of Conjugated Oligomers. Organic Letters 2006, 8, 5243-5246.

(32) Weininger, D. SMILES, a Chemical Language and Information System: 1: Introduction to Methodology and Encoding Rules. Journal of Chemical Information and Computer Sciences 1988, 28, 31-36.

(33) Weininger, D.; Weininger, A.; Weininger, J. L. SMILES. 2. Algorithm for Generation of Unique SMILES Notation. Journal of Chemical Information and Computer Sciences 1989, 29, 97-101.

(34) Weininger, D. Smiles. 3. Depict. Graphical Depiction of Chemical Structures. Journal of Chemical Information and Computer Sciences 1990, 30, 237-243. 
(35) Halgren, T. A. Merck molecular force field. I. Basis, form, scope, parameterization, and performance of MMFF94. Journal of Computational Chemistry 1996, 17, 490-519.

(36) Rappe, A. K.; Casewit, C. J.; Colwell, K. S.; III, W. A. G.; Skiff, W. M. UFF, a full periodic table force field for molecular mechanics and molecular dynamics simulations. Journal of the American Chemical Society 2002, 114, 10024-10035.

(37) O’Boyle, N. M.; Banck, M.; James, C. A.; Morley, C.; Vandermeersch, T.; Hutchison, G. R. Open Babel: An open chemical toolbox. Journal of Cheminformatics 2011, 3,33 .

(38) Bannwarth, C.; Ehlert, S.; Grimme, S. GFN2-xTB - An Accurate and Broadly Parametrized Self-Consistent Tight-Binding Quantum Chemical Method with Multipole Electrostatics and Density-Dependent Dispersion Contributions. Journal of Chemical Theory and Computation 2019, 15, 1652-1671.

(39) Brandenburg, J. G.; Bannwarth, C.; Hansen, A.; Grimme, S. B97-3c: A revised lowcost variant of the B97-D density functional method. The Journal of Chemical Physics 2018, 148, 064104 .

(40) Neese, F. The ORCA program system. Wiley Interdisciplinary Reviews: Computational Molecular Science 2012, 2, 73-78.

(41) Neese, F. Software update: the ORCA program system, version 4.0. Wiley Interdisciplinary Reviews: Computational Molecular Science 2018, 8, e1327.

(42) Systematic optimization of long-range corrected hybrid density functionals. The Journal of Chemical Physics 2008, 128, 084106.

(43) Weigend, F. Accurate Coulomb-fitting basis sets for H to Rn. Physical Chemistry Chemical Physics 2006, 8, 1057-1065. 
(44) O'boyle, N. M.; Tenderholt, A. L.; Langner, K. M. cclib: A library for packageindependent computational chemistry algorithms. Journal of Computational Chemistry 2008, 29, 839-845. 